\title{
Properties of dust in early-type galaxies
}

\author{
M. K. Patil ${ }^{1, \star}$, S. K. Pandey ${ }^{2, \star}$, D. K. Sahu ${ }^{3}$, and A. Kembhavi ${ }^{4}$ \\ 1 School of Physical Sciences, S.R.T.M. University, Nanded 431 606, India \\ e-mail: patil@iucaa.ernet.in \\ 2 School of Studies in Physics, Pt. Ravishankar Shukla University, Raipur 492 010, India \\ e-mail: skp@iucaa.ernet.in \\ 3 Indian Institute of Astrophysics, Bangalore 560 034, India \\ e-mail: dks@crest.ernet.in \\ ${ }^{4}$ Inter University Centre for Astronomy \& Astrophysics (IUCAA), Post Bag 4, Ganeshkhind, Pune 411 007, India \\ e-mail: akk@iucaa.ernet.in
}

Received 25 May 2005 / Accepted 16 August 2006

\section{ABSTRACT}

\begin{abstract}
We report optical extinction properties of dust for a sample of 26 early-type galaxies based on the analysis of their multicolour CCD observations. The wavelength dependence of dust extinction for these galaxies is determined and the extinction curves are found to run parallel to the Galactic extinction curve, which implies that the properties of dust in the extragalactic environment are quite similar to those of the Milky Way. For the sample galaxies, value of the parameter $R_{V}$, the ratio of total extinction in $V$ band to selective extinction in $B$ and $V$ bands, lies in the range 2.03-3.46 with an average of 3.02, compared to its canonical value of 3.1 for the Milky Way. A dependence of $R_{V}$ on dust morphology of the host galaxy is also noticed in the sense that galaxies with a well defined dust lane show tendency to have smaller $R_{V}$ values compared to the galaxies with disturbed dust morphology. The dust content of these galaxies estimated using total optical extinction is found to lie in the range $10^{4}$ to $10^{6} M_{\odot}$, an order of magnitude smaller than those derived from IRAS flux densities, indicating that a significant fraction of dust intermixed with stars remains undetected by the optical method. We examine the relationship between dust mass derived from IRAS flux and the X-ray luminosity of the host galaxies.The issue of the origin of dust in early-type galaxies is also discussed.
\end{abstract}

Key words. galaxies: elliptical and lenticular, cD - galaxies: ISM - ISM : dust, extinction

\section{Introduction}

Recent imaging surveys using both ground and space-based telescopes across the electromagnetic spectrum have amply demonstrated that early-type galaxies, ellipticals (E) and lenticulars (S0) contain complex, multi-phase interstellar medium (ISM). In particular, a large fraction $(\sim 50-80 \%)$ of E-S0 galaxies, at least in our local universe, are now known to possess dust features in a variety of morphological forms, as revealed from high quality optical imaging, thanks to the availability of sensitive array detectors (Goudfrooij et al. 1994a,b; van Dokkum \& Franx 1995; Tran et al. 2001, and references therein). Dust attenuates the radiation at optical wavelengths and re-radiates at infra-red (IR) wavelengths, especially in far-infrared (FIR) bands. FIR emission from early-type galaxies detected using the Infra Red Astronomical Satellite (IRAS) (Knapp et al. 1989), and now with the Infrared Space Observatory (ISO) and Spitzer Space Telescope is attributed to thermal emission from dust having a wide variety of temperatures (Leeuw et al. 2004; Temi et al. 2004; Xilouris et al. 2004). The dust masses derived from ISO data are at least an order of magnitude larger than those estimated using IRAS fluxes, which in turn have been found to be on average an order of magnitude higher than the dust mass estimated using optical extinction.

The study of physical properties of dust such as extinction, reddening and polarization help in deriving important information about the total dust content, size of the dust grains

^ Visiting Associate of IUCAA, Pune, India. responsible for extinction and its variation with environment, metallicity, star formation history and redshift of the host galaxy. Dust properties in galaxies at high redshift are noticeably different from those found at later cosmic times, because the dust grains are smaller in size due to their different formation history (through type II Supernovae) and also due to the short time available for accreting heavy atoms and coagulation with other grains (Maiolino et al. 2004). Thus, detailed study of the dust properties in the extragalactic environment provides useful clues not only for understanding the origin and fate of dust in external galaxies, but also for the subsequent evolution of host galaxies.

Our knowledge of dust properties largely relies on the interaction of dust particles with the electromagnetic radiation i.e., on attenuation and scattering of starlight, collectively known as extinction, and re-radiation by dust at longer wavelengths. The spectral dependence of extinction, termed as extinction curve, is found to depend strongly on the composition, structure and size distribution of the dust grains. Therefore, the first step in understanding the physical properties of dust in the extragalactic environment is to derive the extinction curves for the sample galaxies. For our own Galaxy, the $\lambda$-dependence of extinction is derived by comparing the spectral energy distribution (SED) of a pair of stars of identical spectral and luminosity class with and without dust in front of them (Massa et al. 1983). Any difference in the measured magnitude and colour of these stars is attributed to the dust extinction. The standard extinction law thus derived is found to be uniformly applicable in our Galaxy from optical to near-infrared wavelengths. Over the UV to far-IR wavelength 
range the standard extinction law is characterized by a single parameter $R_{V}$, the ratio of total extinction $A_{V}$ in $V$ band to the selective extinction in $B$ and $V$ bands i.e., $E(B-V)$, with $R_{V}=3.1$ (Savage \& Mathis 1979; Rieke \& Lebofsky 1985; Mathis 1990). However, within the Milky Way, the value of $R_{V}$ is found to vary between 2.1 to 5.6 depending on the line of sight (Valencic et al. 2004). Similarly, the extinction curves have also been derived for a few neighbouring spiral galaxies using this method and the $R_{V}$ value is found to vary considerably for these galaxies (Brosch 1988 ), and even within a galaxy from one location to another.

In the case of external galaxies, as individual stars cannot be resolved, the above method is not applicable; instead, a variety of indirect methods have been proposed to establish the $\lambda$-dependence of the dust extinction, and to estimate $R_{V}$ values. In one of the widely used methods, comparison of light distribution of the original galaxy with its dust-free smooth model gives an estimate of the extinction caused by dust present in the original galaxy. The extinction law for external galaxies can be deduced by performing this exercise at different wavelengths. The absence of spiral arms, H II regions and other inhomogeneities results in a fairly smooth light distribution in earlytype galaxies. This allows one to easily and accurately construct the dust-free model of the galaxy required to study dust extinction properties. Despite this rather convenient situation, dust extinction properties have been investigated only for a handful of galaxies, most notably by Brosch \& Loinger (1991), Brosch \& Almoznino (1997), Goudfrooij et al. (1994a,b) and extended to a few more individual galaxies by Sahu et al. (1998), Dewangan et al. (1999), Falco et al. (1999), Keel \& White (2001), Motta et al. (2002) etc. In all these studies it has been observed that the optical extinction curves in the extragalactic environment closely resemble that of the Milky Way, with $R_{V}$ values comparable to the canonical value of 3.1. Dust being an important component of the ISM, it is essential to extend this kind of analysis to a larger sample of dusty early-type galaxies.

It has been shown that the morphology of dust closely matches that of the ionized gas in a large fraction of galaxies ( 50-80\%) (Goudfrooij et al. 1994a; Ferrari et al. 1999), and in some cases with the X-ray emitting region too (Goudfrooij \& Trinchieri 1998), pointing to a possible physical connection between hot, warm and cold phases of ISM in early-type galaxies. In the multiphase ISM, dust grains can act as an efficient agent in transporting heat from hot gas to cold gas giving rise to the observed warm phase of ISM (Trinchieri et al. 1997). Again a detailed investigation of dust properties is called for.

We have an ongoing program of detailed surface photometric study of a large sample of early-type galaxies containing dust to investigate dust properties in the extragalactic environment and compare them with those of the Milky Way. This paper reports on dust properties in a sample of 26 early-type galaxies, based on their deep, broad band optical (BVRI) imaging observations. Section 2 describes the sample selection, observations and preliminary reduction of the acquired data, Sect. 3 describes the properties of dust for the sample galaxies, in Sect. 4 results obtained are discussed. Our results are summarized in Sect. 5. Through out this paper we assume $H_{0}=50 \mathrm{~km} \mathrm{~s}^{-1} \mathrm{Mpc}^{-1}$.

\section{Observations and data reduction}

We have carried out multiband optical imaging observations of 26 dusty early-type galaxies (11 E and $15 \mathrm{~S} 0$ ) as a part of our ongoing program studying dust properties in a large sample of early-type galaxies taken from Ebneter \& Balick (1985), Véron-Cetty \& Véron (1988), Knapp et al. (1989), van Dokkum \& Franx (1995). The objects were chosen depending on the availability of observing nights and weather conditions, and as such no strict criterion has been applied for the sample selection. Table 1 gives the global parameters such as coordinates, morphological type, heliocentric velocity, luminosity, size and environment of the target galaxies.

Deep CCD images of the program galaxies were obtained using various observing facilities available in India, during December 1998 to August 2003. Table 2 gives details of the instruments used during different observing runs and Table 3 gives the log of observations. Except for the IAO observing run, where Bessel $U, B, V, R, I$ filters were used, observations were made in Johnson $B, V$ and Cousins $R, I$ filters. Generally, exposure times were adjusted so as to achieve roughly equal signal-to-noise (S/N) ratio for a galaxy in different bands. Apart from the object frames, several calibration frames such as bias, twilight sky flats etc. were also taken in each observing run. For photometric calibration of the data, open cluster M67 and standard stars from Landolt's list (Landolt 1992) were observed during photometric nights.

Standard preprocessing steps such as bias subtraction and flat fielding were done using the standard tasks available within IRAF $^{1}$. Multiple frames taken in each filter were geometrically aligned to an accuracy better than one tenth of a pixel by measuring centroids of several common stars in the galaxy frames, and were then combined to improve the $\mathrm{S} / \mathrm{N}$ ratio. This also enabled easy removal of cosmic ray events. The cosmic ray hits that were left after combining the frames were further eliminated using the cosmicrays task in IRAF. Sky background in the galaxy frame was estimated using the box method (see, e.g., Sahu et al. 1998); median of a $5 \times 5$ pixels box at various locations in the frame, generally away from the galaxy and not affected by the stars, was estimated and its mean value was taken as a measure of the sky background which was then subtracted from the respective galaxy frame in the corresponding band. As the total field coverage of the CCDs used was large compared to the optical size of the program galaxies, the box method for sky estimation was found suitable for the present study. Cleaned, sky subtracted $B, V, R, I$ images of individual galaxies were convolved with a Gaussian function to match the seeing of the best frame with that of the worst frame to construct colour-index images of the galaxies.

\section{Properties of dust: the method and results}

Even though the program galaxies were known to contain dust features, we re-examined them using a variety of image processing techniques like quotient image, unsharp masking, colourindex image, etc., to confirm the presence of dust features and their spatial distribution. $(B-V)$ and $(B-R)$ colour index images were constructed using geometrically aligned, seeing matched direct images. $(B-V)$ colour index images of some of the dust lane galaxies are shown in Fig. 1, where brighter shades represent dust occupied redder regions. Our analysis confirms the presence of dust in all the 26 galaxies studied here. Dust is present in variety of forms; five galaxies have multiple dust lanes parallel to the major axis, twelve objects show a well-defined dust lane aligned either along major or minor axes, three galaxies show dust rings or arcs, while others show nuclear dust patches.

1 IRAF is distributed by the National Optical Astronomy Observatories (NOAO), which is operated by the Association of Universities, Inc. (AURA) under co-operative agreement with the National Science Foundation. 
Table 1. Global parameters of the program galaxies.

\begin{tabular}{|c|c|c|c|c|c|c|c|}
\hline $\begin{array}{c}\text { Object } \\
\text { (1) }\end{array}$ & $\begin{array}{c}\mathrm{RA} \\
(\mathrm{J} 2000.0) \\
(2)\end{array}$ & $\begin{array}{c}\text { Dec } \\
(\mathrm{J} 2000.0) \\
(3)\end{array}$ & $\begin{array}{c}\text { Morph. } \\
\text { RC3(RSA) } \\
\text { (4) }\end{array}$ & $\begin{array}{l}B_{T}^{0} \\
(5)\end{array}$ & $\begin{array}{c}V_{\text {helio }} \\
\left(\mathrm{km} \mathrm{s}^{-1}\right) \\
(6)\end{array}$ & $\begin{array}{c}\text { size } \\
\text { (arcmin) } \\
(7)\end{array}$ & $\begin{array}{l}\text { CfA Group } \\
\text { (8) }\end{array}$ \\
\hline NGC 524 & $01: 24: 47$ & $09: 32: 21$ & $\mathrm{~S} 0(S 0 / a)$ & 11.17 & 2509 & $2.8 \times 2.8$ & GH13 (8), dominant in group \\
\hline NGC 984 & $02: 34: 43$ & $23: 24: 46$ & So & 13.35 & 4435 & $3.0 \times 2.0$ & - \\
\hline NGC 1172 & 03:01:36 & $-14: 50: 13$ & $\mathrm{E} 1\left(S 0_{1}\right)$ & 12.56 & 1502 & $2.3 \times 1.8$ & - \\
\hline NGC 1439 & 03:44:50 & $-21: 55: 21$ & $\mathrm{E} 1(E 1)$ & 12.18 & 1568 & $2.5 \times 2.3$ & HG32 (21), Eridanus group \\
\hline NGC 2128 & 06:04:34 & $57: 37: 40$ & S0 & 12.66 & 3226 & $1.5 \times 1.1$ & - \\
\hline NGC 2534 & $08: 12: 54$ & $55: 40: 24$ & E1 & 13.38 & 3732 & $1.4 \times 1.2$ & Isolated galaxy \\
\hline NGC 2563 & $08: 20: 35$ & 21:04:09 & So & 13.01 & 4592 & $2.1 \times 1.5$ & In dense part of Cancer cluster \\
\hline NGC 2672 & $08: 49: 22$ & 19:04:30 & $\mathrm{E} 1(E 2)$ & 12.12 & 4164 & $3.0 \times 2.8$ & Brightest in group, pair with N2673 \\
\hline NGC 2693 & 08:56:59 & $51: 20: 51$ & $\mathrm{E} 3(E 2)$ & 12.61 & 4923 & $2.6 \times 1.8$ & GH39 (4) \\
\hline NGC 2907 & $09: 31: 36$ & $-16: 44: 09$ & $\mathrm{~S} 0 / \mathrm{a}(S 0)$ & 12.35 & 1892 & $1.8 \times 1.1$ & Brightest in group, several dEs companion \\
\hline NGC 2911 & 09:33:46 & 10:09:09 & $\mathrm{S} 0\left(S_{0}\right)$ & 12.25 & 3062 & $4.1 \times 3.2$ & GH47 (6) \\
\hline NGC 3078 & 09:58:24 & $-26: 55: 34$ & $\mathrm{E} 2(E 3)$ & 11.94 & 2283 & $2.5 \times 2.1$ & HG29(4), in cluster \\
\hline NGC 3489 & $11: 00: 18$ & $13: 54: 08$ & $\mathrm{SO}(S 0 / a)$ & 11.15 & 613 & $3.5 \times 2.0$ & GH68 (23)/HG56 (30), Leo grp \\
\hline NGC 3497 & $11: 07: 18$ & $-19: 28: 21$ & So & 13.03 & 3672 & $2.6 \times 1.4$ & In cluster, interacting pair \\
\hline NGC 3585 & 11:13:17 & $-26: 45: 20$ & $\mathrm{E} 6(E / S 0)$ & 10.64 & 1206 & $4.7 \times 2.6$ & - \\
\hline NGC 3599 & $11: 15: 27$ & 18:06:46 & So & 12.70 & 713 & $2.7 \times 2.1$ & GH77 (13) \\
\hline NGC 3665 & $11: 24: 43$ & $38: 45: 47$ & $\mathrm{~S} 0\left(S_{0}\right)$ & 11.69 & 2072 & $2.5 \times 2.0$ & GH79 (4), pair with N3658 \\
\hline NGC 3923 & 11:51:02 & $-28: 48: 23$ & $\mathrm{E} 4(E / S 0)$ & 10.62 & 1487 & $5.9 \times 3.9$ & HG28 (4), at least $7 \mathrm{dEs}$ \\
\hline NGC 4459 & 12:29:00 & $13: 58: 46$ & $\mathrm{SO}\left(S_{0} 0\right)$ & 11.21 & 1154 & $3.5 \times 2.7$ & GH106 (248) \\
\hline NGC 5363 & 13:56:07 & 05:15:19 & $\mathrm{IO}(S 0)$ & - & 1113 & $4.1 \times 2.6$ & HG55 (3), pair with N5364 \\
\hline NGC 5485 & 14:07:11 & 55:00:08 & SO $(S 0)$ & 12.31 & 2107 & $2.3 \times 1.9$ & HG77 (4), pair with N5484, N5486 \\
\hline NGC 5525 & $14: 15: 39$ & 14:17:02 & So & 13.78 & 5569 & $1.4 \times 0.9$ & GH134 (8) \\
\hline NGC 5898 & $15: 18: 13$ & $-24: 05: 49$ & $\mathrm{E} 0(S 0)$ & 11.92 & 2153 & $2.2 \times 2.0$ & $\begin{array}{l}\text { In pair with NGC 5903, } \\
\text { several dEs, dS0s companion }\end{array}$ \\
\hline NGC 5903 & $15: 18: 36$ & $-24: 05: 06$ & $\mathrm{E} 2(E / S 0)$ & 12.20 & 2565 & $2.7 \times 2.1$ & $\begin{array}{l}\text { In pair with NGC } 5898, \\
\text { several dEs, dS0s companion }\end{array}$ \\
\hline NGC 7432 & $22: 58: 06$ & 13:08:00 & $\mathrm{E}$ & 14.41 & 7615 & $1.5 \times 1.2$ & In pair \\
\hline NGC 7722 & $23: 38: 41$ & $15: 57: 15$ & S0 & 13.19 & 4197 & $1.7 \times 1.4$ & In pair with a spiral \\
\hline
\end{tabular}

Notes: Cols. (2) and (3) list galaxy co-ordinates, Col. (4) lists morphological classification of the galaxies, RSA type is given in the parenthesis. The total, corrected blue luminosity of the program galaxies are listed in Col. (5), while Col. (6) lists heliocentric velocity and Col. (7) optical size of the galaxies, all taken from RC3 (de Vaucoulers et al. 1991). Column (8) lists CfA group membership HG or GH listed by Huchra \& Geller (1982; HG82) and Geller \& Huchra (1983; GH83), number in the parenthesis represents number of galaxies in a given group.

Table 2. Details of telescopes and instrumentation.

\begin{tabular}{|c|c|c|c|c|c|c|c|}
\hline Observing run & Dec.98 & Dec.99 & Mar.2k & Mar.01 & Apr.01 & May 03 & Aug.03 \\
\hline Observatory & ARIES & ARIES & VBO & VBO & VBO & IAO & IAO \\
\hline Telescope size(in m) & 1.04 & 1.04 & 2.34 & 2.34 & 2.34 & 2.0 & 2.0 \\
\hline CCD Type & TEK & TEK & Phot & Phot & Phot & Phot & Phot \\
\hline Format (\# pixels) & $1 \mathrm{k} \times 1 \mathrm{k}$ & $2 \mathrm{k} \times 2 \mathrm{k}$ & $1 \mathrm{k} \times 1 \mathrm{k}$ & $1 \mathrm{k} \times 1 \mathrm{k}$ & $1 \mathrm{k} \times 1 \mathrm{k}$ & $1 \mathrm{k} \times 1 \mathrm{k}$ & $1 \mathrm{k} \times 1 \mathrm{k}$ \\
\hline Binning & $2 \times 2$ & $2 \times 2$ & $1 \times 1$ & $1 \times 1$ & $1 \times 1$ & $1 \times 1$ & $1 \times 1$ \\
\hline Scale ("/bin) & 0.73 & 0.73 & 0.72 & 0.72 & 0.72 & 0.285 & 0.285 \\
\hline Field of view & $13^{\prime} \times 13^{\prime}$ & $13^{\prime} \times 13^{\prime}$ & $10^{\prime} \times 10^{\prime}$ & $10^{\prime} \times 10^{\prime}$ & $10^{\prime} \times 10^{\prime}$ & $5^{\prime} \times 5^{\prime}$ & $5^{\prime} \times 5^{\prime}$ \\
\hline
\end{tabular}

Notes: Telescope $-1.04 \doteq 1.04 \mathrm{~m}$ Sampurnanand Telescope of Aryabhatta Research Institute of Observational Sciences (ARIES), Naini Tal; $2.34 \doteq 2.34 \mathrm{~m}$ Vainu Bappu Telescope of V.B. Observatory $(\mathrm{VBO})$, Kavalur; $2.0 \doteq 2.0 \mathrm{~m}$ Himalayan Chandra Telescope of Indian Astronomical Observatory (IAO), Mt. Saraswati, Hanle.

CCD type - TEK: Tektronics, Phot : Photometrics.

NGC 2907 and NGC 7722 have at least four extended dust lanes running parallel to the optical major axis. NGC 5363 has two lanes, the inner one is short and aligned along minor axis, while the other is extended and has an intermediate orientation.

\subsection{Extinction maps}

To estimate the effect of dust extinction, we compare the light distribution in the original galaxy image with its dust free model. As early-type galaxies have a fairly smooth and symmetric stellar light distribution with respect to the nucleus, one can easily construct its smooth, dust free model by fitting ellipses to the isophotes of observed image. This method has been used by a number of researchers (Brosch \& Loinger 1991; Goudfrooij et al. 1994b; Sahu et al. 1998, and references therein) to study extinction properties of dust in the extragalactic environment.

We have fitted ellipses to the isophotes of the observed galaxy images using the ellipse fitting routine available in the STSDAS $^{2}$ package, which is based on a procedure described by Jedrzejewski (1987).

Starting with trial values of ellipticity, position angle, and centre coordinates of the galaxy image, an ellipse was fitted to the isophote at a given semi-major axis length after masking all

2 STSDAS is distributed by the Space Telescope Science Institute, operated by AURA, Inc., under NASA contract NAS 5-26555. 
Table 3. Observing log.

\begin{tabular}{|c|c|c|c|c|c|c|c|c|c|}
\hline \multirow{2}{*}{$\begin{array}{l}\text { Galaxy } \\
\text { (1) }\end{array}$} & \multicolumn{4}{|c|}{ Exposure time (s) } & \multicolumn{4}{|c|}{ Seeing (") } & \multirow{2}{*}{$\begin{array}{c}\text { Notes } \\
\text { (10) }\end{array}$} \\
\hline & $\begin{array}{l}B \\
(2)\end{array}$ & $\begin{array}{l}V \\
(3)\end{array}$ & $\begin{array}{l}R \\
\text { (4) }\end{array}$ & $\begin{array}{l}I \\
(5)\end{array}$ & $\begin{array}{c}B \\
(6)\end{array}$ & $\begin{array}{c}V \\
(7)\end{array}$ & $\begin{array}{c}R \\
(8)\end{array}$ & $\begin{array}{c}I \\
(9)\end{array}$ & \\
\hline NGC 524 & $900(4)$ & $900(3)$ & $400(3)$ & $400(3)$ & 2.65 & 2.41 & 2.14 & 2.11 & $\# 1$ \\
\hline NGC 984 & $900(4)$ & $600(4)$ & $400(3)+200(1)$ & $300(4)$ & 3.01 & 2.72 & 2.71 & 2.91 & \#2 \\
\hline NGC 1172 & $900(4)$ & $900(3)$ & $400(3)$ & $400(3)$ & 2.66 & 2.55 & 2.46 & 2.61 & \#2 \\
\hline NGC 1439 & $600(4)$ & $600(3)$ & $200(4)$ & $200(3)$ & 2.55 & 1.93 & 1.86 & 1.95 & \#2 \\
\hline NGC 2128 & $900(4)$ & $900(3)$ & $400(2)$ & $400(2)$ & 2.81 & 2.46 & 2.39 & 2.09 & \#1 \\
\hline NGC 2534 & $900(4)$ & $900(3)$ & $600(3)$ & $600(3)$ & 2.51 & 2.25 & 1.79 & 2.06 & \#1 \\
\hline NGC 2563 & - & $600(4)$ & $400(3)$ & $400(3)$ & - & 4.98 & 4.52 & 4.32 & \#3 \\
\hline NGC 2672 & $900(4)$ & $600(3)$ & $300(3)+200(1)$ & $300(3)$ & 1.90 & 1.87 & 2.08 & 1.88 & \#2 \\
\hline NGC 2693 & $900(4)$ & $600(3)$ & $360(4)$ & $300(4)$ & 3.30 & 3.33 & 3.07 & 2.96 & \#4 \\
\hline NGC 2907 & $900(4)$ & $600(3)$ & $300(4)$ & $300(3)$ & 3.46 & 2.61 & 2.57 & 2.46 & \#2 \\
\hline NGC 2911 & $1200(3)+600(1)$ & $600(4)$ & $300(5)$ & $300(4)$ & 2.62 & 2.30 & 2.00 & 1.86 & \#1 \\
\hline NGC 3078 & $600(4)$ & - & $300(2)$ & - & 2.87 & - & 3.29 & - & \#4 \\
\hline NGC 3489 & $500(3)$ & $400(3)$ & $400(1)$ & $360(1)$ & 2.97 & 2.75 & 2.77 & 2.87 & \#2 \\
\hline NGC 3497 & - & $700(3)$ & - & $420(2)$ & - & 2.38 & - & 2.41 & \#7 \\
\hline NGC 3585 & $600(4)$ & $600(3)$ & $300(2)$ & - & 3.16 & 3.07 & 2.90 & - & \#3 \\
\hline NGC 3599 & $600(3)$ & $300(3)$ & $300(3)$ & $200(3)$ & 2.59 & 2.54 & 2.47 & 2.21 & \#2 \\
\hline NGC 3665 & $600(3)$ & $400(3)$ & $300(2)$ & - & 2.11 & 1.96 & 1.82 & - & \#6 \\
\hline NGC 3923 & $900(4)$ & $600(4)$ & $300(4)$ & - & 3.24 & 3.60 & 3.19 & - & \#3 \\
\hline NGC 4459 & $900(4)$ & $600(3)$ & $360(4)$ & - & 2.50 & 2.41 & 2.20 & - & \#5 \\
\hline NGC 5363 & $600(4)$ & $600(3)$ & $300(4)$ & $300(2)$ & 2.12 & 1.96 & 2.42 & 1.82 & \#6 \\
\hline NGC 5485 & $600(6)$ & $600(3)$ & $300(4)$ & $300(6)$ & 2.11 & 1.96 & 1.54 & 1.52 & \#7 \\
\hline NGC 5525 & $600(6)$ & $500(4)+280(1)$ & - & - & 2.01 & 2.11 & - & - & \#7 \\
\hline NGC 5898 & $800(3)$ & $400(3)$ & $300(2)$ & $300(2)$ & 3.25 & 2.61 & 3.06 & 2.89 & \#3 \\
\hline NGC 5903 & $800(3)$ & $400(3)$ & $300(2)$ & $300(2)$ & 3.25 & 2.61 & 3.06 & 2.89 & \#3 \\
\hline NGC 7432 & $600(6)$ & $600(3)$ & $300(6)$ & $300(6)$ & 1.41 & 1.37 & 1.25 & 1.48 & \#6 \\
\hline NGC 7722 & $600(5)$ & $600(3)$ & $400(3)$ & $400(3)$ & 1.45 & 1.75 & 1.30 & 1.61 & \#4 \\
\hline
\end{tabular}

Notes to Table 3: Col. (1) lists sample galaxies. Columns (2)-(5) list exposure time in seconds for $B, V, R$, and $I$ bands, respectively, number in parenthesis represents the number of exposures acquired in a given band. Seeing (in arcsec) in different bands are listed in Cols. (6)-(9). Column (10) lists observing run and instruments used; \#1 $\doteq$ ARIES, 1998; \#2 $\doteq$ ARIES, 1999; \#3 $\doteq$ VBO, March 2000; \#4 $\doteq$ VBO, March 2001; \#5 $\doteq$ VBO, April 2001; \#6 $\doteq$ IAO, May 2003; \#7 $\doteq$ IAO, August 2003 (see Table 2).

the obvious regions occupied by foreground stars and interacting galaxies, which were ignored during the ellipse fit. The fitting was continued by incrementing the semi-major axis length by $10 \%$ until signal reaches $3 \sigma$ level of the background, and continued inward until the centre of the galaxy. A model image constructed using the best fit ellipses was then subtracted from the original galaxy image and its residual image was generated. The residual image was examined and regions occupied by dust and other hidden features were flagged and rejected in the next run of ellipse fitting. As the majority of galaxies from this sample contain complex dust lanes or patches passing through their centres, accurate estimation of centre coordinates was not possible. To minimize the error in this estimate, isophote fitting was first carried out for $R$ or $I$ band images, which are least affected by dust extinction among the available bands and centre coordinates were determined by averaging those of the best fitted ellipses. Centre coordinates thus determined were kept fixed in the second run and the above described ellipse-fitting procedure was repeated. The same centre coordinates were used to fit ellipses to the isophotes in other bands.

The "dust free" model of the program galaxies thus generated were used to construct "extinction maps" in magnitude scale using the relation

$A_{\lambda}=-2.5 \times \log \left[\frac{I_{\lambda, \mathrm{obs}}}{I_{\lambda, \text { model }}}\right]$

where $A_{\lambda}$ represents the amount of extinction in a particular band $\lambda(=B, V, R, I)$ while $I_{\lambda \text {,obs }}$ and $I_{\lambda \text {,model }}$ represent ADU count levels in the original and model galaxies, respectively.
Extinction maps for the sample galaxies are shown in Fig. 2, where brighter features represent regions of higher optical depth associated with the dust extinction. Asymmetries seen in the dust features of NGC 5903 and NGC 7432 are due to the masking of foreground stars present near the centre of galaxies.

\subsection{Extinction curves}

The next step involves the quantification of total extinction in each band and deriving the extinction curve. For this purpose, masks were set on the regions occupied by dust in extinction maps generated above, and numerical values of $A_{\lambda}$ (with $\lambda=B, V, R$ and $I$ ) were extracted as the mean extinction within a square box of $5 \times 5$ pixels (comparable to the size of the seeing disk) and the box was slided over the dust-occupied region in each galaxy. To avoid seeing effects, we excluded nuclear regions (radius $\leq 5^{\prime \prime}$ ) of the program galaxies from analysis. The values of extinctions $A_{\lambda}(x, y)$ thus measured at different locations in individual bands were used to estimate the local values of selective extinction or colour excess $E(\lambda-V)=A_{\lambda}-A_{V}$ as a function of position across the dust-occupied region.

A linear regression fit was performed between various local values of total extinction $\left(A_{B}, A_{V}, A_{R}, A_{I}\right)$ and the slopes of the best fits were assigned to be the average slope of $A_{x}$ versus $A_{y}$ (where $x, y=B, V, R$ and $I ; x \neq y$ ) and the reciprocal slope of $A_{y}$ versus $A_{x}$ (Goudfrooij et al. 1994a; Sahu et al. 1998; Dewangan et al. 1999). Likewise, slopes of the fitted lines of the regression for $A_{\lambda}(\lambda=B, V, R$ and $I)$ and $E(B-V)$ were also derived. The best fitting slopes were used to derive $R_{\lambda}\left[\equiv \frac{A_{\lambda}}{E(B-V)}\right]$ 

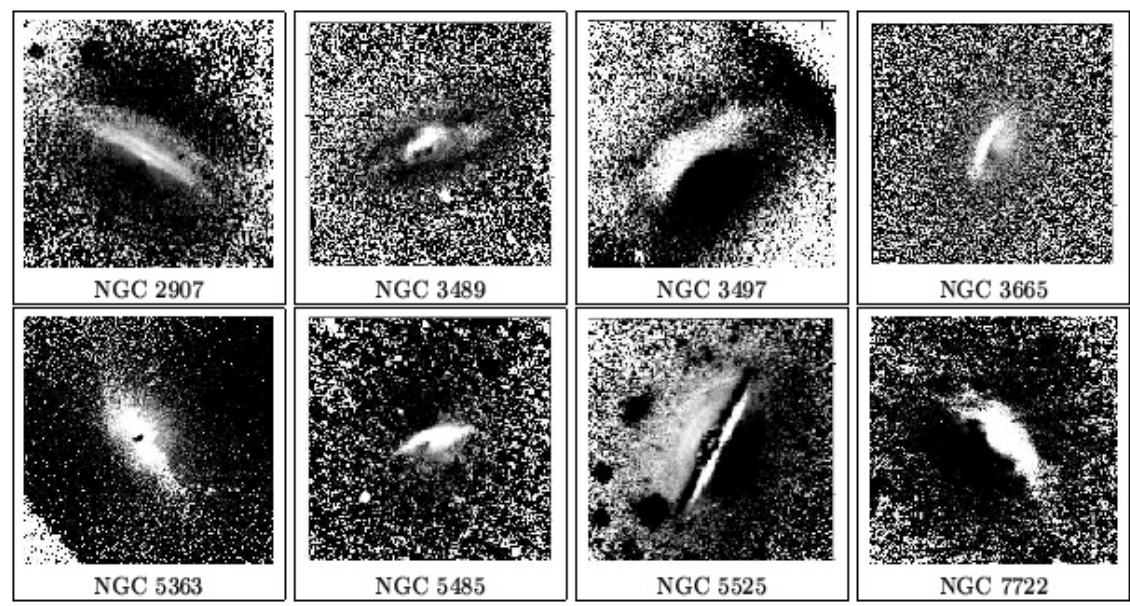

Fig. 1. $(B-V)$ colour index maps of some of the prominent dust lane galaxies; brighter shade represents dust occupied regions.


NGC 2672
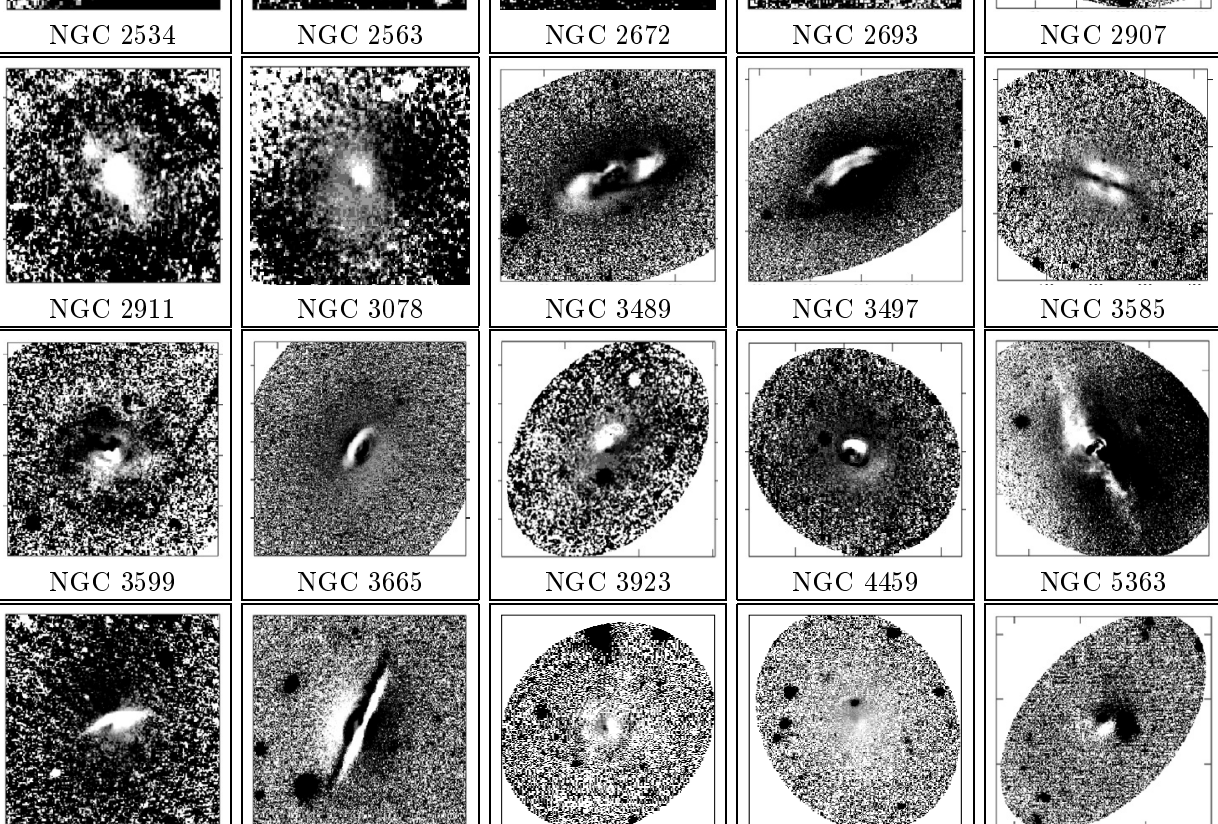

NGC 3078
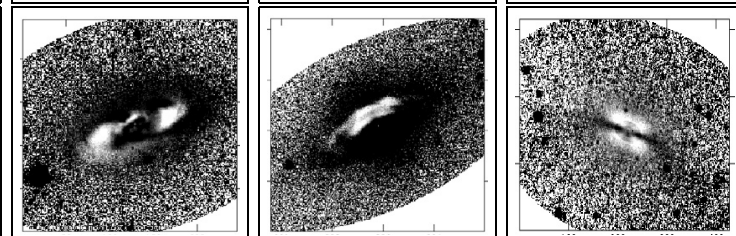

NGC 3489

NGC 3497

NGC 3585
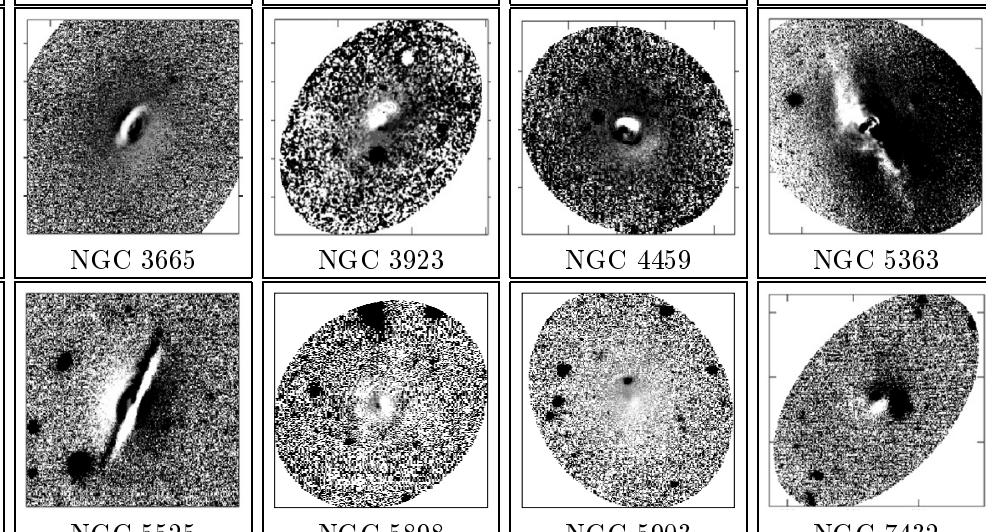

NGC 3923

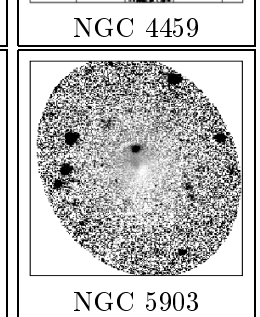

NGC 5363
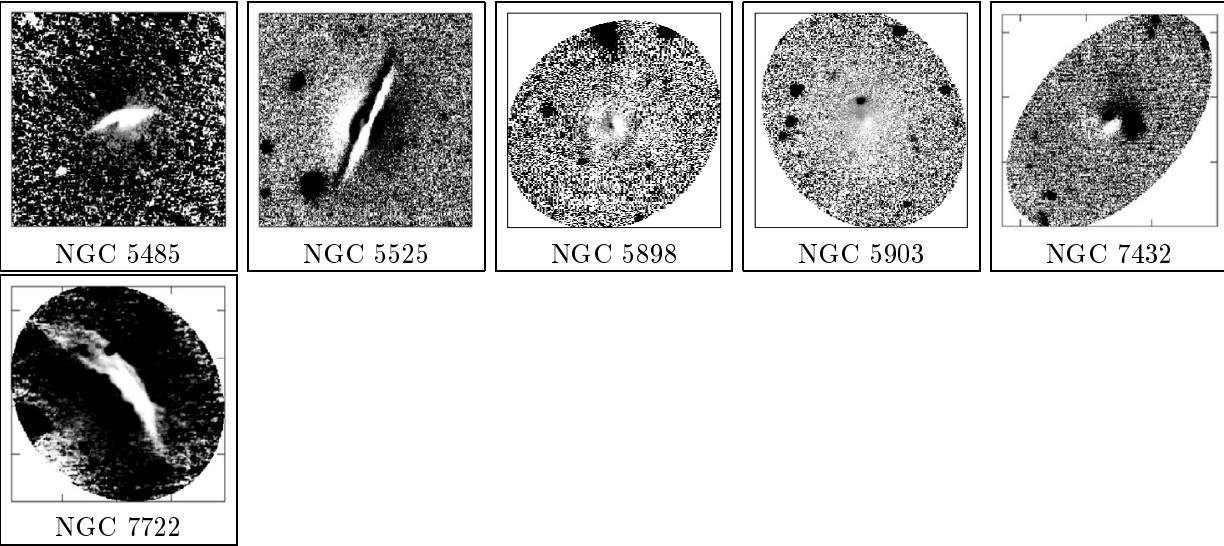

Fig. 2. Extinction maps; brighter shades represent dust occupied regions. North is up and East is to right. 
Table 4. $R_{\lambda}$ values and relative grain sizes.

\begin{tabular}{|c|c|c|c|c|c|}
\hline $\begin{array}{l}\text { Object } \\
\text { (1) }\end{array}$ & $\begin{array}{l}R_{\mathrm{B}} \\
(2)\end{array}$ & $\begin{array}{l}R_{V} \\
\text { (3) }\end{array}$ & $\begin{array}{l}R_{R} \\
\text { (4) }\end{array}$ & $\begin{array}{l}R_{I} \\
(5) \\
\end{array}$ & $\begin{array}{l}\frac{\leq a>}{a_{\text {Gal }}} \\
(6) \\
\end{array}$ \\
\hline NGC 524 & $4.46 \pm 0.39$ & $3.46 \pm 0.16$ & $2.21 \pm 0.47$ & $1.57 \pm 0.45$ & $1.06 \pm 0.02$ \\
\hline NGC 984 & $4.19 \pm 0.36$ & $3.19 \pm 0.21$ & $2.43 \pm 0.19$ & $2.06 \pm 0.17$ & $1.08 \pm 0.08$ \\
\hline NGC 1172 & $4.25 \pm 0.17$ & $3.25 \pm 0.19$ & $2.39 \pm 0.19$ & $1.95 \pm 0.14$ & $1.07 \pm 0.05$ \\
\hline NGC 1439 & $3.45 \pm 0.21$ & $2.45 \pm 0.17$ & $2.01 \pm 0.15$ & $1.76 \pm 0.13$ & $0.93 \pm 0.08$ \\
\hline NGC 2128 & $4.37 \pm 0.28$ & $3.37 \pm 0.27$ & $2.06 \pm 0.29$ & $1.59 \pm 0.30$ & $1.04 \pm 0.06$ \\
\hline NGC 2534 & $3.03 \pm 0.19$ & $2.03 \pm 0.28$ & $1.61 \pm 0.30$ & $1.18 \pm 0.38$ & $0.80 \pm 0.04$ \\
\hline NGC 2563 & - & $3.10 \pm 0.00$ & $2.29 \pm 0.74$ & $1.19 \pm 0.51$ & $0.96 \pm 0.03$ \\
\hline NGC 2672 & $4.27 \pm 0.14$ & $3.27 \pm 0.28$ & $2.48 \pm 0.14$ & $1.77 \pm 0.15$ & $1.06 \pm 0.02$ \\
\hline NGC 2693 & $4.46 \pm 0.23$ & $3.46 \pm 0.26$ & $2.87 \pm 0.45$ & $2.59 \pm 0.36$ & $1.30 \pm 0.04$ \\
\hline NGC 2907 & $3.25 \pm 0.40$ & $2.25 \pm 0.48$ & $1.19 \pm 0.29$ & $0.49 \pm 0.23$ & $0.77 \pm 0.03$ \\
\hline NGC 2911 & $3.84 \pm 0.35$ & $2.84 \pm 0.29$ & $1.98 \pm 0.31$ & $1.13 \pm 0.37$ & $0.92 \pm 0.04$ \\
\hline NGC 3078 & $4.10 \pm 0.17$ & - & $2.39 \pm 0.19$ & - & $1.02 \pm 0.03$ \\
\hline NGC 3489 & $4.38 \pm 0.25$ & $3.38 \pm 0.21$ & $2.64 \pm 0.19$ & $1.70 \pm 0.15$ & $1.09 \pm 0.02$ \\
\hline NGC 3497 & - & $3.10 \pm 0.00$ & - & $1.53 \pm 0.65$ & $1.02 \pm 0.02$ \\
\hline NGC 3585 & $4.45 \pm 0.44$ & $3.45 \pm 0.33$ & $3.00 \pm 0.24$ & - & $1.17 \pm 0.04$ \\
\hline NGC 3599 & $3.49 \pm 0.15$ & $2.49 \pm 0.09$ & $2.12 \pm 0.06$ & $1.57 \pm 0.14$ & $0.93 \pm 0.06$ \\
\hline NGC 3665 & $4.29 \pm 0.20$ & $3.29 \pm 0.13$ & $2.33 \pm 0.23$ & - & $1.03 \pm 0.02$ \\
\hline NGC 3923 & $4.16 \pm 0.43$ & $3.16 \pm 0.39$ & $2.03 \pm 0.16$ & - & $0.98 \pm 0.04$ \\
\hline NGC 4459 & $3.94 \pm 0.24$ & $2.94 \pm 0.18$ & $2.26 \pm 0.16$ & - & $0.90 \pm 0.02$ \\
\hline NGC 5363 & $3.78 \pm 0.27$ & $2.78 \pm 0.16$ & $2.18 \pm 0.36$ & $1.89 \pm 0.28$ & $0.92 \pm 0.04$ \\
\hline NGC 5525 & $4.15 \pm 0.29$ & $3.15 \pm 0.17$ & - & - & $0.99 \pm 0.01$ \\
\hline NGC 5898 & $4.15 \pm 0.30$ & $3.15 \pm 0.46$ & $2.46 \pm 0.29$ & $1.84 \pm 0.41$ & $1.04 \pm 0.03$ \\
\hline NGC 5903 & $4.07 \pm 0.30$ & $3.07 \pm 0.22$ & $2.28 \pm 0.39$ & $2.01 \pm 0.34$ & $1.05 \pm 0.08$ \\
\hline NGC 7432 & $3.89 \pm 0.28$ & $2.89 \pm 0.21$ & $2.46 \pm 0.19$ & $1.94 \pm 0.22$ & $1.05 \pm 0.09$ \\
\hline NGC 7722 & $3.89 \pm 0.25$ & $2.89 \pm 0.20$ & $2.03 \pm 0.14$ & $1.78 \pm 0.10$ & $0.94 \pm 0.02$ \\
\hline NGC 5485 & $\begin{array}{cc}R_{U} & R_{\mathrm{B}} \\
4.38 \pm 0.24 & 3.65 \pm 0.21 \\
\end{array}$ & $\begin{array}{c}R_{V} \\
2.65 \pm 0.17\end{array}$ & $\begin{array}{c}R_{R} \\
1.86 \pm 0.12\end{array}$ & $\begin{array}{c}R_{I} \\
1.40 \pm 0.18\end{array}$ & $0.92 \pm 0.04$ \\
\hline The Galaxy & 4.10 & 3.10 & 2.27 & 1.86 & 1.00 \\
\hline
\end{tabular}

Notes on Table 4: Cols. (2) to (5) lists the $R_{\lambda}\left[\equiv \frac{A_{\lambda}}{E(B-V)}\right]$ values with $\lambda=B, V, R$, and $I$. Column (6) lists the relative (mean) grain size in the program galaxies with respect to that responsible for the Galactic curve. NGC 5485 represents data in five bands $(U, B, V, R$ and $I)$. For comparison, Galactic $R_{\lambda}$ values are listed in the last row.

for the dust occupied regions in the program galaxies and are listed in Table 4 along with their associated errors. $R_{\lambda}$ values for the Milky Way taken from Rieke \& Lebofsky (1985) are also listed in the table for comparison.

The extinction curves for the program galaxies are given in Fig. 3 along with that for the Milky Way. These figures demonstrate that the extinction curves for the majority of galaxies run parallel to that of the Galactic curve, except for a few cases (NGC 1439, 2534, 2693, 3585, 5363) for which we see "concave" extinction curves. The fact that, on average, extinction curves for the sample galaxies are parallel to that of our Galaxy implies that the dust extinction properties in the extragalactic environment are similar to those of the Milky Way. However, $R_{V}$ for program galaxies is found to be different from the canonical value of 3.1 for the Milky Way, as evident from Table 4.

Assuming that the chemical composition of dust grains in the extragalactic environment is similar to that of our Galaxy, smaller (larger) $R_{V}$ values imply that the dust grains responsible for interstellar reddening are smaller (larger) than the grains in our Galaxy. This allows one to compute the relative grain size in the sample galaxies by making use of available models of chemical composition and shape of the dust grains. Out of several "dust models" a two-component model consisting of spherical graphite and silicate grains, with an adequate mixture of sizes (Mathis et al. 1977) is able to explain the observed extinction curves in the Milky Way as well as in the Local Group (e.g.,
Clayton et al. 2003). This model assumes uncoated refractory particles having a power law size distribution $n(a)=n_{0} a^{-3.5}$, where $a$ represents grain radius. As the present study is restricted to the optical regime, the model proposed by Mathis et al. (1977) serves as a good approximation for this purpose.

Figure 3 shows that $R_{\lambda}\left[\equiv \frac{A_{\lambda}}{E(B-V)}\right]$ varies linearly with the inverse of the wavelength and is consistent with the result $Q_{\text {ext }} \propto$ $\lambda^{-1}$ expected for small grains i.e., for $x \leq 1$, where $x=\frac{2 \pi a}{\lambda}$; $a$ is the grain radius and $Q_{\text {ext }}$ is the extinction efficiency factor. Thus, for a given $Q_{\text {ext }}$ value, the mis-match between the extinction curve for the Milky Way and those for the program galaxies is attributed to the difference in grain size between the program galaxies and the Milky Way. Therefore, one can estimate the relative grain size in such cases by shifting the observed extinction curve along $\lambda^{-1}$ axis until it best matches the Galactic extinction curve (cf. Goudfrooij et al. 1994b), in the sense that the extinction curve lying below (above) the Galactic curve will correspond to smaller (larger) grain size relative to that of Galactic dust grains. The relative grain sizes thus derived for the sample galaxies are listed in Col. 6 of Table 4.

\subsection{Dust mass estimation}

We have estimated dust content of the program galaxies using (i) optical extinction studied here and (ii) IRAS fluxes at $60 \mu \mathrm{m}$ 


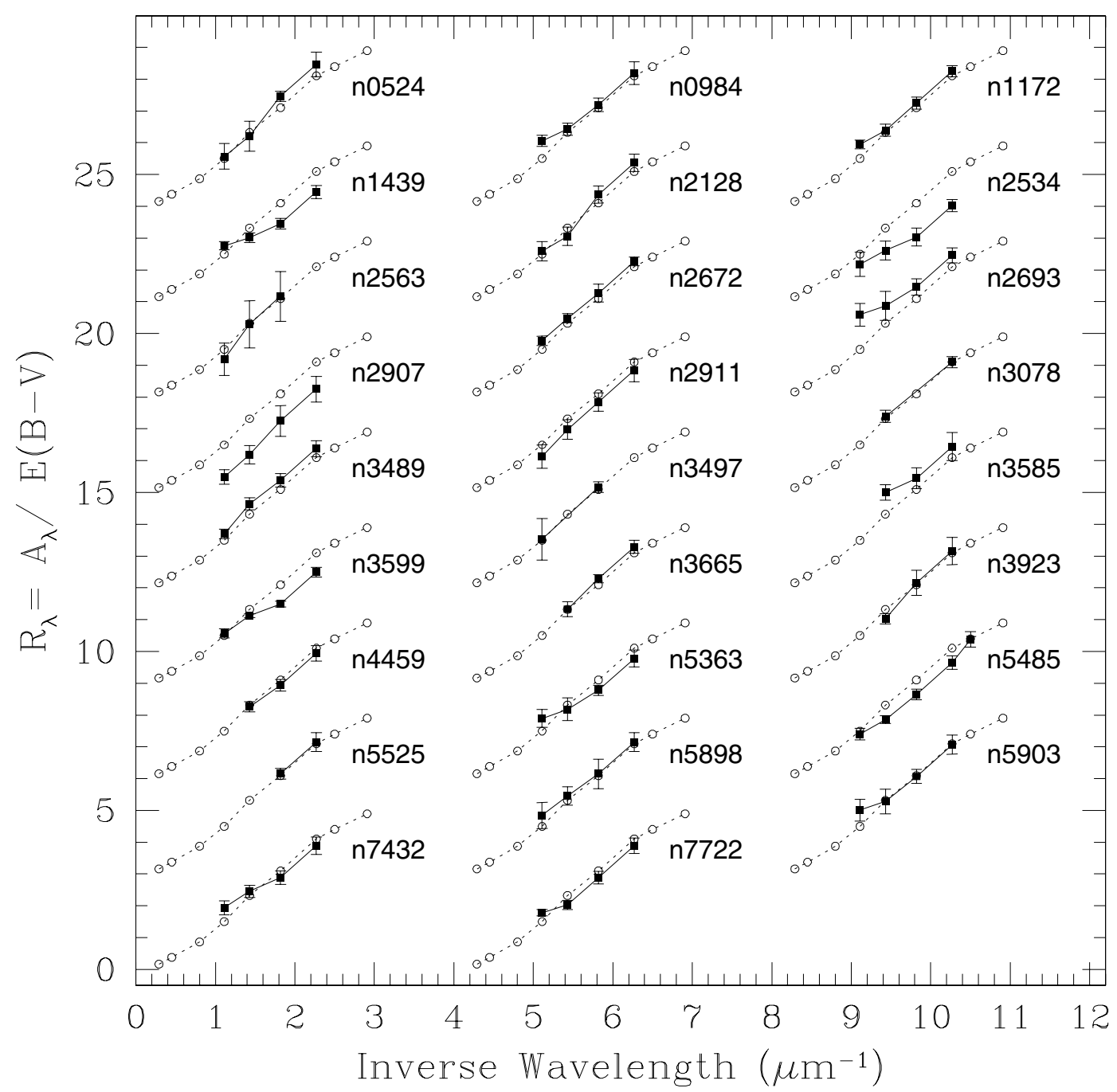

Fig. 3. Extinction curves for the program galaxies (filled squares, solid lines) along with the canonical curve for the Galaxy (open circles, dashed lines) for comparison. The offset (shift of the curves along the $X$ or $Y$ axis in order to separate them) for the $X$-axis is 4 , that for the $Y$-axis is 3 .

and $100 \mu \mathrm{m}$ taken from Knapp et al. (1989), as described in the following subsections.

\subsubsection{Using optical extinction}

To estimate dust mass using total optical extinction, we followed the method described by Goudfrooij et al. (1994b), which is outlined here. For a given grain size distribution function $n(a)$ of the spherical grains of radius $a$ and extinction efficiency $Q_{\text {ext }}(a, \lambda)$, the extinction cross-section at wavelength $\lambda$ is given by

$C_{\text {ext }}(\lambda)=\int_{a_{-}}^{a_{+}} Q_{\text {ext }}(a, \lambda) \pi a^{2} n(a) \mathrm{d} a$

where $a_{-}$and $a_{+}$are the lower and upper cutoffs of the grain size distribution, respectively. Assuming $n(a)$ to be the same over the entire dusty region and using the definition of the efficiency factor, $Q_{\text {ext }}(a, \lambda)=C_{\text {ext }}(a, \lambda) / \pi a^{2}$ (ratio of extinction cross-section to the geometrical cross-section), the total extinction due to dust at wavelength $\lambda$ is expressed as

$A_{\lambda}=1.086 C_{\text {ext }}(\lambda) \times l_{\mathrm{d}}$ where $l_{\mathrm{d}}$ is the dust column length along the line of sight. The column length density in units of $\mathrm{g} \mathrm{cm}^{-2}$ for the dust is then expressed as

$\Sigma_{\mathrm{d}}=\int_{a_{-}}^{a_{+}} \frac{4}{3} \pi a^{3} \rho_{\mathrm{d}} n(a) \mathrm{d} a \times l_{\mathrm{d}}$

where $\rho_{\mathrm{d}}$ gives the specific grain mass density which is taken to be $\sim 3 \mathrm{~g} \mathrm{~cm}^{-3}$ for graphite and silicate grains (Draine \& Lee 1984). This is then multiplied by the total area occupied by dust to obtain the dust mass, $M_{\mathrm{d}}=\Sigma_{\mathrm{d}} \times$ Area, expressed in solar mass units.

The measured total extinction in $V$ band $\left(A_{V}\right)$ can be used to compute dust mass using the size distribution of Mathis et al. (1977) as

$$
n(a)=n_{0} a^{-3.5} \quad\left(a_{-} \leq a \leq a_{+}\right)
$$

where $a_{-}=0.005 \mu \mathrm{m}$ and $a_{+}=0.22 \mu \mathrm{m}$ for the Milky Way with $R_{V}=3.1$ (cf. Draine \& Lee 1984). Since the observed extinction curves in the program galaxies refer to the dust grains at the upper end of the size distribution (Goudfrooij et al. 1994b), upper limits of the grain size in the target galaxies were scaled accordingly using the relation

$a_{+}=\frac{\langle a\rangle}{a_{\mathrm{Gal}}} \times 0.22 \mu \mathrm{m}$ 
where $\frac{\langle a\rangle}{a_{\mathrm{Gal}}}$ is the relative grain size for the program galaxies and are listed in Table 4.

Further, to estimate the extinction efficiency of the dust grains we assume spherical grains composed of silicate and graphite with nearly equal abundance (see, Mathis et al. 1977). The values of the extinction efficiency for silicate and graphite grains are taken as

$Q_{\text {ext,silicate }}= \begin{cases}0.8 a / a_{\text {silicate }} & \text { for } a<a_{\text {silicate }} \\ 0.8 & \text { for } a \geq a_{\text {silicate }},\end{cases}$

$Q_{\text {ext,graphite }}= \begin{cases}2.0 a / a_{\text {graphite }} & \text { for } a<a_{\text {graphite }} \\ 2.0 & \text { for } a \geq a_{\text {graphite }}\end{cases}$

with $a_{\text {silicate }}=0.1 \mu \mathrm{m}$, and $a_{\text {graphite }}=0.05 \mu \mathrm{m}$. Using these parameters and the dust column density, the total dust mass $\left(M_{\mathrm{d} \text {,optical }}\right)$ contained in the program galaxies was estimated. In determining the mean visual extinction, we included all those regions with $\tau_{V} \geq 0.02$. The computed dust masses for the sample galaxies using total optical extinction $\left(M_{\mathrm{d}, \text { optical }}\right)$ are given in Col. 6 of Table 5.

\subsubsection{Using IRAS densities}

Using IRAS flux densities measured at 60 and $100 \mu \mathrm{m}$, we first calculate the dust grain temperature in the program galaxies using relation, $T_{\mathrm{d}}=49\left(\frac{S_{60}}{S_{100}}\right)^{0.4}$ (Young et al. 1989). The dust content $\left(M_{\mathrm{d}, \mathrm{IRAS}}\right)$ in the sample galaxies was then computed using the relation (Hildebrand 1983):

$M_{\mathrm{d}}=\frac{4}{3} a \rho_{\mathrm{d}} D^{2} \frac{F_{v}}{Q_{v} B_{v}\left(T_{\mathrm{d}}\right)}$

where $a, \rho_{\mathrm{d}}$ and $D$ are the grain radius, specific grain mass density and distance of the galaxy, respectively. $F_{v}, Q_{v}$ and $B_{v}\left(T_{\mathrm{d}}\right)$ are the observed flux density, grain emissivity and the Planck function of temperature $T_{\mathrm{d}}$ at frequency $v$, respectively. We adopted $\rho_{\mathrm{d}}=3 \mathrm{~g} \mathrm{~cm}^{-3}$ and $\frac{4 a \rho_{\mathrm{d}}}{3 Q_{v}}=0.04 \mathrm{~g} \mathrm{~cm}^{-2}$ for $0.1 \mu \mathrm{m}$ grains at $100 \mu \mathrm{m}$ (Hildebrand 1983). The derived dust masses from IRAS flux densities are listed in Col. 5 of the Table 5. Due to the fact that IRAS was insensitive to the dust emitting at wavelengths longer than $100 \mu \mathrm{m}$ (i.e., dust cooler than about $20 \mathrm{~K}$ ), these estimates of dust masses using IRAS flux densities represent the lower limits (Tsai \& Mathews 1996).

\section{Discussion}

- Extinction curves derived for the sample galaxies reveal that the $R_{V}$ value lies in the range between 2.03 to 3.46 for ellipticals, and 2.25 to 3.46 for lenticulars, with an average of 3.01, compared to 3.1 in the case of the Milky Way. For some of the sample galaxies the $R_{V}$ value is close to the classical Galactic value, while for some of them the $R_{V}$ value is significantly less than 3.1. Interestingly, all galaxies (e.g., NGC 2534, 2907, 2911, 5363, 5485 and 7722) showing smaller $R_{V}$ values exhibit well-settled dust lane or lanes with an average $R_{V}$ equal to 2.80 , implying that the "larger" grains responsible for the optical extinction are roughly $25 \%$ "smaller" in size compared to the grains responsible for the extinction curve in the Milky Way. For a sample of 10 elliptical galaxies, Goudfrooij et al. (1994b) arrived at $R_{V}$ in the range of 2.1 to 3.3 , with characteristic dust grains up to $30 \%$ smaller in size compared to the canonical grains in the
Milky Way. Thus, our results are in good agreement with those of Goudfrooij et al. (1994b). For a few of the remaining galaxies (e.g., NGC 524, 2672, 2693, 3489, 3585) extinction curves are found to lie slightly above the Galactic extinction curve, implying marginally larger values of $R_{V}$, and these are found to have ring or arc shaped dust morphologies. Thus, our results seems to indicate that the galaxies having $R_{V}$ values smaller than the canonical value (and hence smaller grains) exhibit smooth, regularly distributed dust lanes, whereas the galaxies with larger $R_{V}$ values exhibit irregular dust morphologies (Goudfrooij et al. 1994b).

- Dust masses derived from optical extinction are found to lie in the range $\sim 10^{4}$ to $10^{6} M_{\odot}$ and are in good agreement with the earlier estimates for the early-type galaxies (Brosch et al. 1990; Goudfrooij et al. 1994b; Sahu et al. 1998; Ferrari et al. 1999; Dewangan et al. 1999; Tran et al. 2001; Patil et al. 2002). The dust masses derived using the optical methods depend on the assumption of a foreground screen in front of the background light. Therefore, this method is not sensitive to any dust embedded or intermixed with the stars within the galaxy and hence the optical method always provides a lower limit of the true dust content of the host galaxy. Comparison of the dust masses derived from IRAS flux densities and optical extinctions reveals that $M_{\mathrm{d}, \text { IRAS }}$ is larger than $M_{\text {d,optical }}$ by a factor of 7.8 for the sample of galaxies studied here. Goudfrooij \& de Jong (1995) for a sample of elliptical galaxies alone reported this ratio to be equal to 8.4. In the case of S0 galaxies, as pointed out by Sahu et al. (1998) and Dewangan et al. (1999), this mass discrepancy is less significant compared to that for ellipticals. Using ISO data Temi et al. (2004) estimated a dust content of NGC 1172 and NGC 5363 of $9.9 \times 10^{5} M_{\odot}$ and $2.00 \times 10^{6} M_{\odot}$, respectively, these estimates are roughly an order of magnitude larger than our estimates from IRAS fluxes. This in turn implies that although ISO data acts as a more reliable tracer of the true dust content in the extragalactic environment, it renders the dust mass discrepancy even more significant than using IRAS flux densities.

- There are several processes that may alter the effective dust grain size in the external galaxies, like destruction of grains due to sputtering in supernova blast waves, grain-grain collisions, sputtering by thermal ions (warm and hot) etc., as has been discussed by Goudfrooij (1999). But, which one of these would work as the dominant process for destruction of grains leading to smaller grain size depends on the conditions of specific galaxies. In particular, for galaxies immersed in hot X-ray emitting gas, sputtering by hot ions is expected to be the dominant process for destruction and alteration of the grain size. In order to investigate this point further we collected X-ray flux for the sample galaxies from the literature, and examined the relationship between the dust mass and X-ray luminosity, both normalized to the blue luminosity. The result shown in Fig. 4a reveals a marginal anticorrelation suggesting that high $\left(L_{\mathrm{X}} / L_{\mathrm{B}}\right)$ galaxies harbour less dust, in agreement with the predictions of Goudfrooij et al. (1994b).

- The issue of the origin of dust in early-type galaxies is rather controversial. Suggested sources by which galaxies may acquire dust include (i) internal: in the form of mass-loss from evolved stars distributed throughout the galaxy more or less uniformly, and (ii) external: in which a gas-rich companion donates gas and dust either via direct merger, accretion or tidal capture. Dwek \& Scalo (1980) have demonstrated that evolved red giant stars are the dominant source of dust 
Table 5. Dust properties.

\begin{tabular}{|c|c|c|c|c|c|c|c|c|}
\hline Object & Dust morphology & IRAS & $\overline{\mathrm{Ix}(\mathrm{mJy})}$ & $\overline{\log \left(M_{\text {dIRAS }}\right)}$ & $\bar{L} \log \left(M_{\text {dop }}\right.$ & $\overline{T_{\mathrm{d}}}$ & $\overline{\mathcal{M}}$ & $\tau_{c}$ \\
\hline (1) & (2) & $\begin{array}{r}60 \pm \text { err } \\
\text { (3) }\end{array}$ & $100 \pm$ err & $\begin{array}{c}\left(\mathrm{M}_{\odot}\right) \\
(5)\end{array}$ & $\begin{array}{l}\left(M_{\odot}\right) \\
(6)\end{array}$ & $\begin{array}{l}(\mathrm{K}) \\
(7)\end{array}$ & $\begin{array}{c}\left(M_{\odot} \mathrm{yr}^{-1}\right) \\
(8)\end{array}$ & $\begin{array}{c}\left(10^{6} \mathrm{yr}\right) \\
(9)\end{array}$ \\
\hline NGC 524 & dust rings & $780 \pm 34$ & $1820 \pm 114$ & $5.95 \pm 0.11$ & $4.91 \pm 0.14$ & $35 \pm 4$ & 1.06 & 7.8 \\
\hline NGC 984 & maj. dust lane & $140 \pm 37$ & $120 \pm 106$ & $4.85 \pm 0.88$ & $4.50 \pm 0.07$ & $<30$ & $<0.89$ & 5.5 \\
\hline NGC 1172 & nucl. dust & $0 \pm 41$ & $0 \pm 72$ & $<4.35$ & $4.16 \pm 0.18$ & - & $<0.12$ & 13.5 \\
\hline NGC 1439 & min. dust lane & $0 \pm 32$ & $300 \pm 35$ & $<5.80$ & $5.43 \pm 0.19$ & $\leq 20$ & $<0.04$ & 23.0 \\
\hline NGC 2128 & min.dust lane & $870 \pm 37$ & $2160 \pm 166$ & $6.22 \pm 0.11$ & $4.42 \pm 0.12$ & $34 \pm 3$ & 0.10 & - \\
\hline NGC 2534 & min.dust lane & $380 \pm 50$ & $800 \pm 184$ & $5.71 \pm 0.28$ & $4.10 \pm 0.18$ & $36 \pm 4$ & 0.15 & - \\
\hline NGC 2563 & min. dust lane & $0 \pm 27$ & $0 \pm 187$ & $<4.70$ & $3.75 \pm 0.15$ & - & $<0.51$ & 3.3 \\
\hline NGC 2672 & nucl. dust & $0 \pm 45$ & $440 \pm 45$ & $<6.30$ & $5.30 \pm 0.30$ & $\leq 20$ & $<0.69$ & - \\
\hline NGC 2693 & min dust lane & $210 \pm 37$ & $790 \pm 114$ & $6.09 \pm 0.20$ & $4.84 \pm 0.15$ & $29 \pm 4$ & 0.02 & 5.8 \\
\hline NGC 2907 & multi. lanes & $310 \pm 26$ & $1090 \pm 194$ & $5.70 \pm 0.21$ & $5.05 \pm 0.22$ & $30 \pm 2$ & 0.47 & - \\
\hline NGC 2911 & maj. dust lane & $290 \pm 28$ & $560 \pm 79$ & $5.59 \pm 0.19$ & $5.54 \pm 0.14$ & $38 \pm 5$ & 0.10 & 8.8 \\
\hline NGC 3078 & nucl. dust & - & - & - & $3.91 \pm 0.08$ & - & - & 11.0 \\
\hline NGC 3489 & dust clouds & - & - & - & $3.94 \pm 0.26$ & - & - & 18.5 \\
\hline NGC 3497 & multi. lanes & 278 & $<1347$ & $<6.49$ & $5.08 \pm 0.14$ & $\leq 26$ & - & - \\
\hline NGC 3585 & nucl. & $160 \pm 42$ & $0 \pm 70$ & $<4.24$ & $3.73 \pm 0.25$ & $\leq 30$ & 0.37 & 14.7 \\
\hline NGC 3599 & min. dust lane & - & - & - & $3.73 \pm 0.23$ & - & - & 24.0 \\
\hline NGC 3665 & maj. dust lane & $1960 \pm 40$ & $6690 \pm 163$ & $6.60 \pm 0.07$ & $5.02 \pm 0.28$ & $30 \pm 3$ & 0.19 & 23.1 \\
\hline NGC 3923 & nucl. dust & $0 \pm 35$ & $0 \pm 120$ & $<4.70$ & $4.46 \pm 0.08$ & - & 0.61 & 10.6 \\
\hline NGC 4459 & dust ring & $1920 \pm 67$ & $4280 \pm 119$ & $5.71 \pm 0.12$ & $3.66 \pm 0.14$ & $36 \pm 8$ & 0.48 & 23.2 \\
\hline NGC 5363 & multi. lanes & $1700 \pm 46$ & $4450 \pm 45$ & $5.72 \pm 0.18$ & $5.05 \pm 0.14$ & $33 \pm 13$ & 0.01 & 23.1 \\
\hline NGC 5485 & min. dust lane & $150 \pm 34$ & $850 \pm 88$ & $6.01 \pm 0.17$ & $4.36 \pm 0.11$ & $25 \pm 4$ & 0.16 & 13.0 \\
\hline NGC 5525 & maj. lane + ring & - & - & - & $5.99 \pm 0.10$ & - & - & - \\
\hline NGC 5898 & min dust lane & $130 \pm 37$ & $200 \pm 64$ & $4.85 \pm 0.41$ & $4.48 \pm 0.07$ & $41 \pm 9$ & 0.25 & 5.4 \\
\hline NGC 5903 & nucl. dust & $0 \pm 23$ & $0 \pm 112$ & $<6.31$ & $4.05 \pm 0.08$ & - & 0.43 & 5.2 \\
\hline NGC 7432 & min. dust lane & - & - & - & $3.98 \pm 0.09$ & - & - & 5.8 \\
\hline NGC 7722 & multi. lanes & $820 \pm 34$ & $2840 \pm 159$ & $6.82 \pm 0.08$ & $6.19 \pm 0.16$ & $30 \pm 2$ & 1.72 & - \\
\hline
\end{tabular}

Notes to Table 5: Col. (2) lists morphology of dust: dust ring(s) - face on dust ring(s); maj.dust lane - dust lane along the optical major axis; min. dust lane - dust lane aligned along minor axis; multi. lanes - multiple dust lanes; dust clouds - dust distribution in the form of clouds; nucl. dust - nuclear dust. Columns (3) and (4) list IRAS flux densities at $60 \mu \mathrm{m}$ and $100 \mu \mathrm{m}$ (taken from Knapp et al. 1989). Columns (5) and (6) list dust masses derived from IRAS flux densities $\left(M_{\mathrm{d}, \mathrm{IRAS}}\right)$ and optical extinction values $\left(M_{\mathrm{d}, \mathrm{optical}}\right)$, respectively. Column (7) gives dust temperature $\left(T_{\mathrm{d}}\right)$, while Col. (8) lists the present day mass-loss rate from the evolved, red-giant stars in the program galaxies. Column (9) lists life time of dust grains derived using Eq. (8).

injection into the ISM of the early-type galaxies. To check the significance of internal sources, using the present day mass-loss rate we estimated the total amount of dust accumulated over the lifetime of a galaxy under the assumption that the properties of the evolved, red-giant stars in the Galactic bulge closely resemble those in elliptical galaxies. For most of the $\mathrm{K}$ and early $\mathrm{M}$ giant stars with effective surface temperatures about $4500 \mathrm{~K}$ and with no circumstellar material, one finds $S_{12 \mu \mathrm{m}} / S_{2.2 \mu \mathrm{m}}=0.08$ (Knapp et al. 1992), while for the stars with circumstellar matter (as in elliptical galaxies) this ratio is 0.13 , about $38 \%$ larger than expected from stars in the Galactic bulge (Knapp et al. 1992). This excess flux measured at $12 \mu \mathrm{m}$ in elliptical galaxies is attributed to the emission from the circumstellar shells produced by mass-loss from the evolved stars, and thus provides a reasonable estimate for the total mass-loss. Following Knapp et al. (1992), we adopt the following relation for the massloss rate:

$\dot{\mathcal{M}}=6 \times 10^{-6}\left(\frac{D}{\mathrm{Mpc}}\right)^{2}\left(\frac{S_{12}-0.042 S_{100}}{\mathrm{mJy}}\right) M_{\odot} \mathrm{yr}^{-1}$.

The term $0.042 S_{100}$ gives the correction to the excess flux at $12 \mu \mathrm{m}$ emission due to contamination from the interstellar dust heated to an equilibrium temperature of about $20 \mathrm{~K}$ by the ambient ultraviolet interstellar radiation field. The inferred values of the mass-loss rates in the sample galaxies are listed in Col. 8 of Table 5.
- Dust injected into the ISM by the evolved stars through mass-loss is also destroyed simultaneously by a variety of processes. In the absence of hot, X-ray emitting gas, the life time of the $0.1 \mu \mathrm{m}$ refractory grains against sputtering through low velocity shock waves or grain-grain collision is $\sim 10^{9} \mathrm{yr}$, while that in the presence of hot coronal gas $\left(T_{\mathrm{e}} \sim 10^{7} \mathrm{~K}\right)$ against collision with the hot protons and $\alpha$-particles is of the order of $\sim 10^{6}-10^{7} \mathrm{yr}$ (cf. Draine \& Salpeter 1979; Goudfrooij \& de Jong 1995). A majority of the galaxies from the present sample are detected as X-ray emitters, and therefore, we estimate the lifetime of the dust grains (Col. 9 of Table 5) of radius $a$ against such collision using the relation

$$
\tau_{\mathrm{d}}=a\left|\frac{\mathrm{d} a}{\mathrm{~d} t}\right|^{-1} \simeq 2 \times 10^{4}\left(\frac{\mathrm{cm}^{-3}}{n_{\mathrm{H}}}\right)\left(\frac{a}{0.01 \mu \mathrm{m}}\right) \mathrm{yr}
$$

(Draine \& Salpeter 1979), where $n_{\mathrm{H}}$ is the proton density in the plasma and $a$ is the grain size. Here $n_{\mathrm{H}}=0.83 n_{\mathrm{e}}$, $n_{\mathrm{e}}$ being the electron density estimated using

$n_{\mathrm{e}}(r)=n_{\mathrm{e}}(0)\left[1+\left(\frac{r}{a_{x}}\right)^{2}\right]^{-3 / 4}$

and

$n_{\mathrm{e}}(0)=0.061\left(\frac{L_{\mathrm{X}}}{10^{41} \mathrm{erg} \mathrm{s}^{-1}}\right)^{1 / 2}\left(\frac{a_{x}}{1 \mathrm{kpc}}\right)^{-3 / 2} \mathrm{~cm}^{-3}$ 

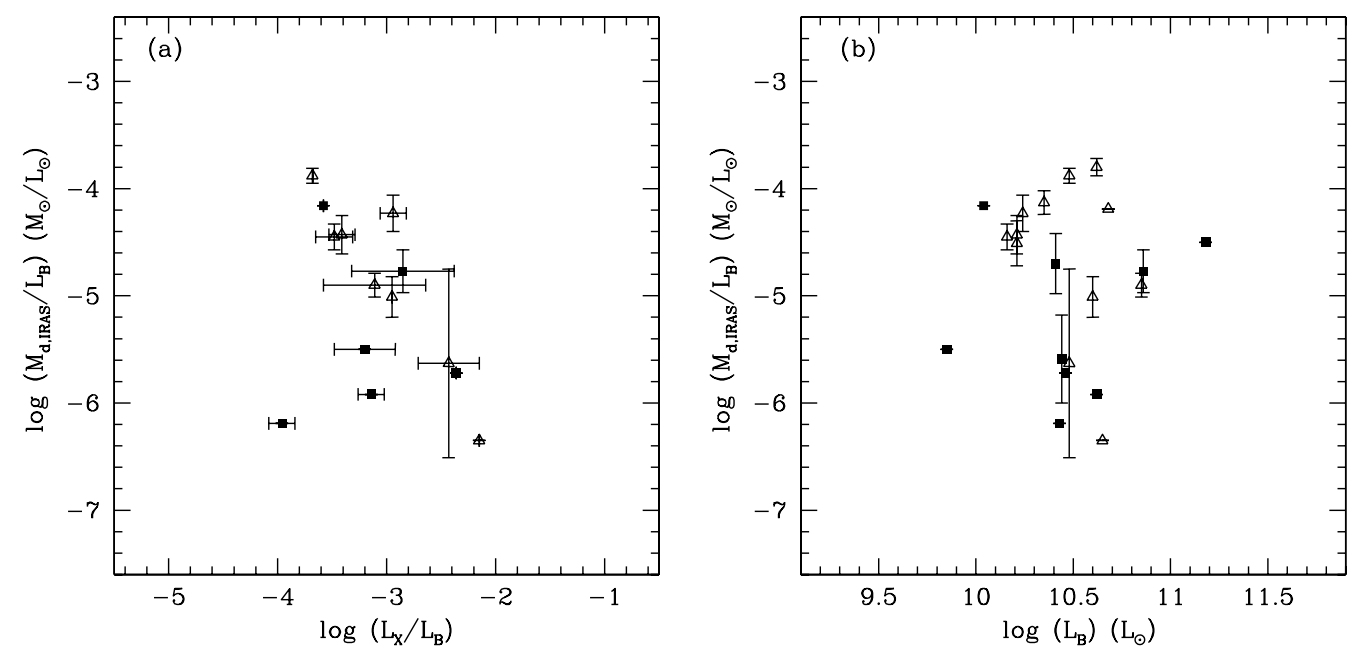

Fig. 4. The ratio of dust mass to blue luminosity $\left(\frac{M_{\mathrm{d}, \mathrm{IRAS}}}{L_{\mathrm{B}}}\right)$ as a function of a) ratio of the X-ray-to-blue luminosity, and b) blue luminosity (filled squares are for E, while open triangles for S0).

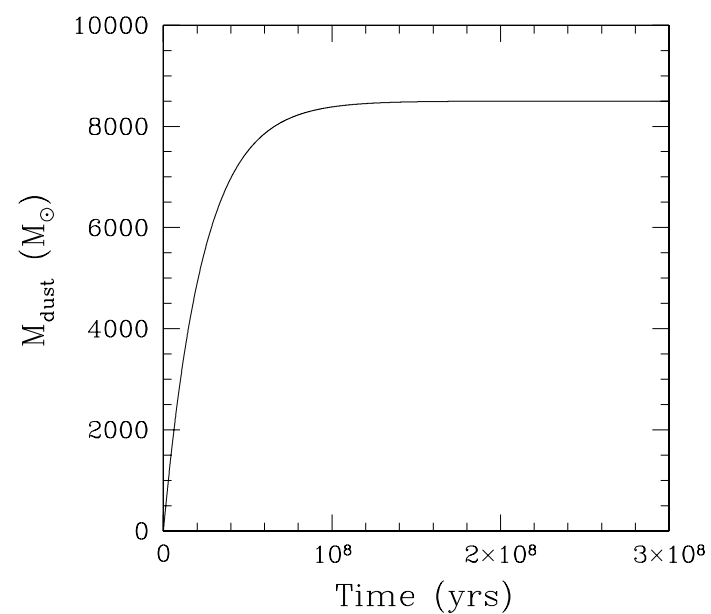

Fig. 5. Total build up of dust mass within NGC 5363 over its life time.

where $n_{\mathrm{e}}(0)$ is the central number density over the X-ray core radius $a_{\mathrm{X}}\left(=1.0\left(\frac{L_{\mathrm{B}}}{10^{11} L_{\odot}}\right)^{0.8} \mathrm{kpc}\right)$ (see, e.g., Canizares et al. 1987, for details). X-ray luminosities reported by Bettoni et al. (2003) were used to derive these parameters. For the grains of radius $0.1 \mu \mathrm{m}$ this time scale is found to lie in the range between $2.4 \times 10^{7} \mathrm{yr}$ and $3.3 \times 10^{6} \mathrm{yr}$ which correspond to NGC 3599 and NGC 2563 having the lowest and highest $\mathrm{X}$-ray luminosities, respectively, in the present sample.

For illustration, we examine the accumulation of dust for the case of NGC $5363\left(\log L_{\mathrm{X}}=40.38 \mathrm{erg} \mathrm{s}^{-1}, n_{\mathrm{H}}=2.2 \times\right.$ $10^{-3} \mathrm{~cm}^{-3}, \tau_{\mathrm{d}}=2.3 \times 10^{7} \mathrm{yr}$ and $\dot{\mathcal{M}}=10^{-2} M_{\odot} \mathrm{yr}^{-1}$ and gas-to-dust ratio of about 100), taking into account the two competing processes, namely mass-loss in the form of dust from the evolved stars and destruction of dust. The rate of accumulation of dust is

$\frac{\partial M_{\mathrm{d}}(t)}{\partial t}=\frac{\partial M_{\mathrm{d}, \mathrm{s}}}{\partial t}-M_{\mathrm{d}}(t) \tau_{\mathrm{d}}^{-1}$

where $\frac{\partial M_{\mathrm{d}, \mathrm{s}}}{\partial t}$ is the rate of mass loss in the form of dust from red giant stars and $\tau_{\mathrm{d}}^{-1}$ is the destruction rate. Integrating this dust accumulation rate over time, the total build up of dust mass in NGC 5363 over its life time is shown in Fig. 5 and is found to be equal to $8.5 \times 10^{3} M_{\odot}$, which is about a factor of 100 times smaller than the observed dust mass. For other galaxies, where X-ray flux was available in the literature, comparison of the build up mass and the observed mass shows a similar discrepancy except for NGC 3585 and NGC 3923, for which the two estimates are roughly equal. This in turn implies that internal sources are not sufficient to account for the observed dust and therefore an external source plays a dominant role in the supply of dust into the ISM of early-type galaxies.

- There are several other indicators that support the external origin of dust in the early-type galaxies. If it is of internal origin, it should exhibit the same global rotational dynamics as that of the stellar system (Kley \& Mathews 1995). However, kinematical studies of dust lane galaxies have shown that the angular momentum vectors of the interstellar gas are often orthogonal to those of the stars, implying that the ISM must have a different origin (Caon et al. 2000). Another good argument in favour of an external, merger-related origin of dust in early-type galaxies is the lack of significant correlation between the $L_{\text {FIR }}$ or dust mass ( $M_{\text {d,IRAS }}$ ) and optical luminosity $\left(L_{\mathrm{B}}\right)$, Fig. $4 \mathrm{~b}$. This suggests that at least some of our sample galaxies have acquired dust externally in a mergerlike event. Similar conclusions were also arrived at by Forbes (1991), Goudfrooij \& de Jong (1995), Temi et al. (2004).

- To explore the possibility that dust could originate through a direct merger or accretion or tidal capture of gas from their encounter with neighbouring galaxies, we searched the environment of the program galaxies and found that the majority of the galaxies are either a part of a group/cluster, or form interacting pairs with their neighbours. Relevant details on the environment of the galaxies taken from Huchra \& Geller (1982), Geller \& Huchra (1983) and Garcia (1993) are given in the Col. 8 of Table 1. Following Tran et al. (2001), we also examined the relationship between the dust content and environment of the program galaxies using parameters like group membership $(N)$ and group harmonic radius $\left(R_{\mathrm{H}}\right)$; $R_{\mathrm{H}}$ provides a rough measure of the size of the group (see, Tran et al. 2001, for details). The group membership and group harmonic radius is plotted as a function of dust mass in Fig. 6. The scatter plots between $M_{\mathrm{d}}$ vs. $N$ and $R_{\mathrm{H}}$ indicate the absence of any significant correlation between dust mass and the two quantities. As group membership in these catalogs mostly relies on the radial velocity measurement 


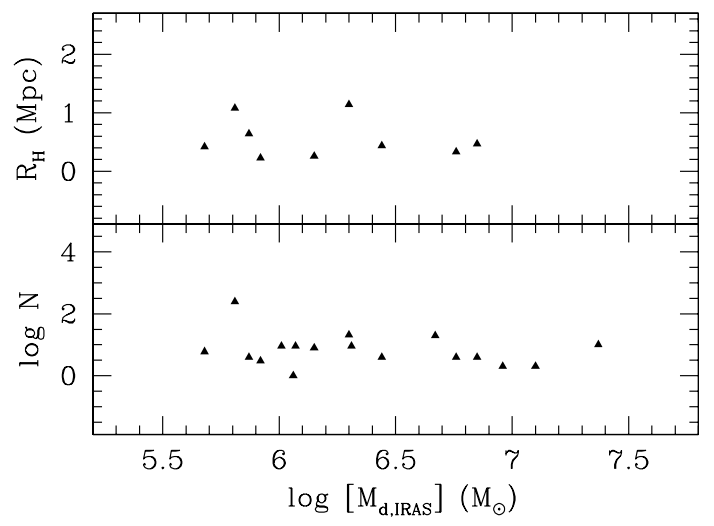

Fig. 6. Environmental dependence of dust mass; upper and lower panels represent dependence on group members $(N)$ and group harmonic radius $\left(R_{\mathrm{H}}\right)$, respectively

of high-luminosity galaxies, small (dwarf) galaxies are often missing from these catalogs, and hence $\log N$ may not be representative of the real situation. If the donors of the dust are supposed to be galaxies similar to those present in the group catalogs mentioned above, this would make the possibility of dust originating from neighbouring companions through merger or tidal capture more complicated. However, one should interpret these results with caution, as a given dusty galaxy might have acquired dust sufficiently long ago that no signature of merger or interaction is visible at the present epoch (Tran et al. 2001).

\section{Conclusions}

We have reported properties of dust in a sample of 26 early-type galaxies based on their multicolour imaging observations. Our results are summarized here.

Extinction curves derived for galaxies studied here run parallel to the canonical curve of the Milky Way, implying that properties of dust in the extragalactic environment are similar to those of the canonical grains in the Milky Way. The $R_{V}$ value, which characterizes the extinction curve in the optical region, is found to vary in the range 2.03-3.46 with an average of 3.02, and is not very much different from the canonical value of 3.1 for our Galaxy. However, several galaxies studied here exhibit smaller $R_{V}$ values suggesting that the "large" grains responsible for the optical extinction are significantly smaller in size than the canonical grains in the Milky Way. Our results indicate that $R_{V}$ values largely depend on the morphology of dust in the host galaxies, in the sense that galaxies with well-defined dust lanes exhibit much smaller grains compared to those with irregular dust morphologies.

The dust content derived by the optical extinction method for the program galaxies is found to lie in the range $10^{4}$ to $10^{6} M_{\odot}$ and is, in general, less than the dust mass derived from IRAS flux densities. This discrepancy is more significant in the case of ellipticals than lenticulars, in good agreement with the results obtained by other researchers for early-type galaxies.

Galaxies with well settled dust lanes contain high dust masses and are weak X-ray sources, in agreement with the prediction of Goudfrooij (1994b). Further, galaxies with wellsettled dust lanes have smaller values of $R_{V}$, and are found to lie in denser environments.

For origin of dust in early-type galaxies, internal mass-loss from evolved stars alone cannot account for the observed dust in these galaxies and therefore points towards an external origin for the dust in these galaxies.

Acknowledgements. The authors thank Drs. Ram Sagar, Vijay Mohan, Mahendra Singh and the staff of ARIES, VBO, IAO for their help during the observing runs. We are grateful to the anonymous referee whose insightful comments helped us to improve the paper. We also acknowledge the enlightening discussion with Dr. Paul Goudfrooij during this work. M.K.P. and S.K.P. are grateful to IUCAA for hospitality and for use of their computational and library facilities. M.K.P. thanks Padmakar, Sudhanshu, Ravi Kumar and Laxmikant Chaware for their valuable help and discussion during this work. We acknowledge the use of NASA/IPAC Extragalactic Database (NED).

\section{References}

Bettoni, D., Galletta, G., \& Burillo, S. G. 2003, A\&A, 405, 5

Brosch, N. 1988, in Dust in the universe (Cambridge Univ. Press), 501

Brosch, N., \& Loinger, F. 1991, A\&A, 249, 327

Brosch, N., \& Almoznino, E. 1997, ApLC, 35, 371

Brosch, N., Almoznino, E., Greenberg, J. M., \& Grosbel, P. G. 1990, A\&A, 233, 341

Canizares, C. R., Fabbiano, G., \& Trinchieri, G. 1987, ApJ, 312, 503

Caon, N., Macchetto, D., \& Pastoriza, M. 2000, ApJS, 127, 39

Clayton, G. C., Wolff, M. J., Sofia, U. J., Gordon, K. D., \& Misselt, K. A. 2003, ApJ, 588, 871

de Vaucouleurs, G., de Vaucouleurs, A., Corwin, H. G., et al. 1991, Third Reference Catalog of bright galaxies (New York: Springer) (RC3)

Dewangan, G. C., Singh, K. P., \& Bhat, P. N. 1999, AJ, 118, 785

Draine, B. T., \& Salpeter, E. 1979, ApJ, 231, 77

Draine, B. T., \& Lee, H. M. 1984, ApJ, 285, 89

Dwek, E., \& Scalo, J. M. 1980, ApJ, 239, 193

Ebneter, K., \& Balick, B. 1985, AJ, 90, 183

Falco, E. E., Impey, C. D., Kochanek, C. S., et al. 1999, ApJ, 523, 617

Ferrari, F., Pastoriza, M. G., Macchetto, F., \& Caon, N. 1999, A\&AS, 136, 269

Forbes, D. A. 1991, MNRAS, 249, 779

Garcia, A. M. 1993, A\&AS, 100, 47

Geller, M. J., \& Huchra, J. P. 1983, ApJS, 52, 61

Goudfrooij, P. 1999, ASP Conf. Ser., ed. M. Valtonen, \& C. Flynn, Proc. IAU Coll., 174

Goudfrooij, P., \& de Jong, T. 1995, A\&A, 298, 784

Goudfrooij, P., \& Trinchieri, G. 1998, A\&A, 330, 123

Goudfrooij, P., Hansen, L., Joregensen, H. E., \& Norgaard-Nielsen, H. U. 1994a, A\&ASS, 105, 341

Goudfrooij, P., de Jong, T., Hansen, L., \& Norgaard-Nielsen, H. U. 1994b, MNRAS, 271, 833

Hildebrand, R. H. 1983, QJRAS, 24, 267

Huchra, J. P., \& Geller, M. J. 1982, ApJ, 257, 423

Jedrzejewski, R. I. 1987, MNRAS, 226, 747

Keel, W. C., \& White, R. E. 2001, AJ, 121, 1442

Kley, W., \& Mathews, W. G. 1995, ApJ, 438, 100

Knapp, G. R., Guhathakurta, P., Kim, D.-W., \& Jura, M. 1989, ApJS, 70, 329

Knapp, G. R., Gunn, J. E., \& Wynn-Williams, C. G. 1992, ApJ, 399, 76

Landolt, A. U. 1992, AJ, 104, 340

Leeuw, L. L., Sanson, A. E., Robson, E. I., Haas, M., \& Kuno, N. 2004, ApJ, 612,837

Maiolino, R., Schneider, R., Oliva, E., Bianchi, S., et al. 2004, Nature, 431, 533

Massa, D., Savage, B., \& Fitzpatrick, E. L. 1983, ApJ, 266, 662

Mathis, J. S. 1990, ARA\&A, 28, 37

Mathis, J. S., Rumpl, W., \& Nordsieck, K. H. 1977, ApJ, 217, 425

Motta, V., Mediavilla, E., Mutoz, J. A., et al. 2002, ApJ, 574, 719

Patil, M. K., Sahu, D. K., Pandey, S. K., et al. 2002, Bull. Astr. Soc. India, 30, 759

Rieke, G. H., \& Lebofsky, M. J. 1985, ApJ, 288, 618

Sahu, D. K., Pandey, S. K., \& Kembhavi, A. K. 1998, A\&A, 333, 803

Savage, B. D., \& Mathis, J. S. 1979, ARA\&A, 17, 73

Temi, P., Brighenti, F., Mathews, W. G., \& Bregman, J. D. 2004, ApJS, 151, 237

Tran, H. D., Tsvetanov, Z., Ford, H. C., \& Davis, J. 2001, AJ, 121, 2928

Trinchieri, G., Noris, L., \& di Serego Alighieri, S. 1997, A\&A, 326, 565

Tsai, J. C., \& Mathews, W. G. 1996, ApJ, 468, 571

Valencic, L., Clayton, G. C., \& Gordon, K. D. 2004, ApJ, 616, 912

Véron-Cetty, M. P., \& Véron, P. 1988, A\&A, 204, 28

van Dokkum, P. G., \& Franx, M. 1995, AJ, 286, 415

Xilouris, E. M., Madden, S. C., Galliano, F., Vigroux, L., \& Sauvage, M. 2004, A\&A, 416, 41

Young, J. S., Xie, S., Kenney, J. D. P., \& Rice, W. L. 1989, ApJS, 70, 699 